\title{
NOUVELLE
}

\section{Contrôle de la persistance des cellules souches neurales des mammifères}

Philippe Taupin

$>$ Les cellules souches neurales sont multipotentes, c'est-à-dire qu'elles peuvent donner naissance aux principaux types cellulaires présents dans le cerveau: neurones, astrocytes et oligodendrocytes, et sont aussi capables de s'autorenouveler [1]. Ces cellules sont à l'origine de la formation du système nerveux central (SNC) au cours du développement embryonnaire. Chez les mammifères adultes, des études récentes ont confirmé la production de nouveaux neurones dans deux régions du SNC: le gyrus denté de l'hippocampe, impliqué dans les processus de mémorisation, et la région sous-ventriculaire, bordant les cavités du cerveau ou ventricules. Les précurseurs neuronaux produits dans la région sous-ventriculaire migrent vers le bulbe olfactif, où ils se différencient en neurones matures $(\rightarrow)$.

Ces travaux ont remis en $(\rightarrow) \mathrm{m} / \mathrm{s} \quad$ cause le dogme selon 2004, $n^{\circ}$ 6-7, lequel nous naissons avec p. 620 un nombre limité de neurones, sans possibilité pour notre cerveau d'en produire de nouveaux à l'âge adulte. Si toutefois l'existence et l'origine de cellules souches neurales reste à démontrer chez l'adulte [2], la fonction des nouveaux neurones produits dans le cerveau adulte chez le rongeur fait actuellement l'objet d'intenses recherches, et l'on a décrit leur implication dans des processus comme la mémoire [3], la dépression [4], et l'odorat [5]. Mais surtout, cette capacité de renouvellement neuronal a fait naître beaucoup d'espoir pour une utilisation à des fins thérapeutiques dans de nombreuses maladies neurodégénératives, comme les maladies d'Alzheimer et de Parkinson.

La détermination de la fonction des cellules souches neurales chez l'adulte, et leur utilisation thérapeutique nécessitent, outre leur caractérisation, la connaissance des mécanismes contrôlant la permanence de ce compartiment et la détermination/différenciation des cellules souches dans les lignées gliales et neuronales. Dans un article publié récemment dans la revue Nature [6], le Dry. Shi et ses collaborateurs ont identifié un rôle pour le récepteur nucléaire TLX dans la persistance et la prolifération des cellules souches neurales adultes: TLX permettrait à ces cellules de rester dans un état indifférencié en réprimant leur différenciation vers un phénotype glial.

TLX est un récepteur nucléaire dit orphelin. Les récepteurs nucléaires orphelins représentent une famille de récepteurs putatifs dont les ligands naturels ne sont pas identifiés. Ces récepteurs contrôlent l'expression de gènes cibles en s'associant à des séquences spécifiques, dites consensus, de ces gènes [7]. Chez la souris, TLX est exprimé au cours du développement exclusivement dans le cerveau, et son expression chute à la naissance [8]. Chez l'adulte, TLX est exprimé faiblement dans l'ensemble du cerveau, et fortement dans les régions neurogéniques $[6,8]$. L'invalidation du gène entraîne, chez les souris knock-out, qui naissent sans phénotype apparent, des troubles comporte-
National Neuroscience

Institute, Singapore,

11 Jalan Tan Tock Seng, 308433 Singapore.

Department of Obstetrics

and Gynaecology, National

University of Singapore.

philippe_taupin@nni.com.sg

mentaux à l'âge adulte, et leur cerveau est de petite taille, avec en particulier un faible développement des régions neurogéniques [9]. Ces observations ont conduit le Dry. Shi et ses collaborateurs à suspecter que TLX pourrait être impliqué dans les mécanismes de la neurogenèse chez l'adulte. Pour démontrer cette hypothèse, les auteurs ont tenté de répondre à deux questions: est-ce que les cellules exprimant TLX ont les caractéristiques de cellules souches neurales, c'est-à-dire multipotentialité et autorenouvellement? Est-ce que la neurogenèse chez l'adulte est affectée par l'absence du gène codant pour TLX?

Les auteurs ont produit deux lignées de souris transgéniques pour le gène $t \mid x$ : des souris hétérozygotes ${ }^{+/-}$qui expriment la protéine TLX, et des souris homozygotes pour la délétion qui n'expriment plus TLX. Chez ces souris, un gène marqueur $(\operatorname{lac} Z)^{1}$ a été substitué au gène $t l x$, ce qui permet de détecter par fluorescence les cellules ainsi modifiées, puisque lacZ est sous le contrôle du promoteur $t / x$. Chez les souris hétérozygotes pour la délétion, les cellules du cerveau adulte co-exprimant TLX et la $\beta$-galactosidase expriment aussi la nestine,un marqueur de l'ensemble des cellules neurales indifférenciées, cellules souches et progéniteurs $[2,10]$. Les critères requis pour qu'une cellule soit qualifiée de cellule souche neurale sont les suivants: il faut démontrer qu'une cellule unique isolée in vitro est multipotente,

1. Le gène lac $Z$ code pour la $\beta$-galactosidase, une enzyme dont l'activité peut être mesurée par des substrats fluorophores. 
c'est-à-dire peut donner naissance aux trois principaux types cellulaires du cerveau, et s'autorenouvelle, perpétuant ainsi les propriétés de multipotentialité [11]. Nous avons isolé, à partir des hémisphères cérébraux de souris adultes hétérozygotes $t x^{+/-}$et homozygotes $t x^{-/-}$, par tri cellulaire, des cellules exprimant la $\beta$-galactosidase et nous avons analysé les propriétés de cellules individuelles. À l'échelon individuel, ces cellules isolées à partir de souris $t l x^{+/-}$sont multipotentes et peuvent s'autorenouveler, contrairement aux cellules isolées à partir de souris déficientes $t / x^{-/-}$qui ne prolifèrent pas, et se différencient en astrocytes. L'expression de TLX est donc requise pour l'expression de la fonction et des caractéristiques de cellules souches neurales.

Pour caractériser le rôle exact de TLX au cours de la neurogenèse chez l'adulte, nous avons analysé la présence de cellules en phase proliférative dans le cerveau de souris $t / x^{-/-}$, après administration de BrdU (bromodésoxyuridine), un analogue de la thymidine, qui s'insère dans l'ADN des cellules au cours de la phase $S$ du cycle cellulaire. Le nombre de cellules BrdU (+) est très faible dans les cerveaux de souris $t / x^{-\prime}$, et nous y avons détecté très peu de cellules progénitrices - exprimant la nestine -, et un nombre plus élevé d'astrocytes. Ainsi, TLX est essentiel au maintien d'une neurogenèse active chez l'adulte.

L'ensemble de ces résultats montrent que la protéine TLX est nécessaire à la permanence du caractère indifférencié et des capacités de prolifération des cellules souches et progénitrices neurales adultes, par la répression de leur différenciation vers le phénotype glial. De fait, la réexpression, in vitro, du gène $t / x$ dans les cellules homozygotes $t / x^{-/-}$ exprimant la $\beta$-galactosidase, induit leur prolifération, et leur autorenouvellement. Quant au mécanisme moléculaire par lequel agit TLX, nous faisons I'hypothèse selon laquelle il s'agit d'une fonction répressive: en effet, des séquences consensus pour TLX ont été identifiées sur les promoteurs de gènes spécifiquement exprimés par les astrocytes, comme la GFAP (glial fibrillar acid protein). Nous suggérons que TLX, en s'associant à ces séquences, inhibe la différenciation des cellules vers le lignage glial; les cellules conservant alors les propriétés des cellules indifférenciées. Cette hypothèse est corroborée par nos observations faites in vitro que les cellules déficientes pour TLX $\left(t / x^{-/-}\right)$ se différencient en astrocytes, et in vivo, que les cerveaux de souris $t / x^{-/-}$présentent un nombre plus élevé de cellules gliales.

Ainsi les résultats présentés permettent à $y$. Shi et à ses collaborateurs non seulement d'attribuer au facteur TLX un rôle de régulation transcriptionelle au cours de la neurogenèse chez l'adulte, mais aussi de mieux en comprendre les mécanismes. TLX jouerait ainsi un rôle majeur au cours de la neurogenèse chez l'adulte, et apparemment un rôle moins important au cours du développement du SNC. Des travaux récents montrent que les gènes de la famille tlx sont impliqués dans la détermination du phénotype de certaines populations de cellules neuronales au cours du développement [12]. Ainsi, les gènes tlx jouent un rôle différentiel dans la maturation du SNC lors du développement, et chez l'adulte. Cette particularité semble différencier TLX d'autres facteurs, comme les facteurs de transcription bHLH, les ligands des récepteurs Notch (Jagged et Delta), et les principaux gènes cibles de la famille HES (hairy enhancer of split), cibles directes de la voie Notch, qui participent à la régulation de la neurogenèse au cours du développement, et chez l'adulte $[13,14]$. Ces données fondamentales permettront peut-être aux chercheurs de mieux maîtriser dans le futur l'utilisation des cellules souches neurales à des fins thérapeutiques. $\diamond$ TXL controls the maintenance of stem cells in the brain

RÉFÉRENCES
1. Gage FH. Mammalian neural stem cells. Science $2000 ; 287$ : 1433-8.

2. Taupin P, Gage FH. Adult neurogenesis and neural stem cells of the central nervous system in mammals. J Neurosci Res 2002; 69: 745-9.

3. Shors TJ, Miesegaes G, Beylin A, et al. Neurogenesis in the adult is involved in the formation of trace memories. Nature 2001; 410: 372-6.

4. Santarelli L, Saxe M, Gross C, et al. Requirement of hippocampal neurogenesis for the behavioral effects of antidepressants. Science 2003; 301: 805-9.

5. Rochefort C, Gheusi G, Vincent JD, Lledo PM. Enriched odor exposure increases the number of newborn neurons in the adult olfactory bulb and improves odor memory. J Neurosci 2002 ; 22: 2679-89.

6. Shi Y, Chichung Lie D, Taupin P, et al. Expression and function of orphan nuclear receptor TLX in adult neural stem cells. Nature 2004; 427: 78-83.

7. Giguere V. Orphan nuclear receptors: from gene to function. Endocrinol Rev 1999; 20: 689-725.

8. Monaghan AP, Grau E, Bock D, Schutz G. The mouse homolog of the orphan nuclear receptor tailless is expressed in the developing forebrain. Development 1995; 121: 839-53.

9. Monaghan AP, Bock D, Gass P, et al. Defective limbic system in mice lacking the tailless gene. Nature 1997; 390: 515-7.

10. Lendahl U, Zimmerman LB, McKay RD. CNS stem cells express a new class of intermediate filament protein. Cell 1990; 60: 585-95.

11. Taupin P, Ray J, Fischer WH, et al. FGF-2-responsive neural stem cell proliferation requires $\mathrm{CCg}$, a novel autocrine/paracrine cofactor. Neuron 2000; 28: 385-97.

12. Cheng L, Arata A, Mizuguchi R, et al. TIx3 and TIx] are post-mitotic selector genes determining glutamatergic over GABAergic cell fates. Nat Neurosci 2004; 7 : 510-7.

13. Hitoshi S, Alexson T, Tropepe V, et al. Notch pathway molecules are essential for the maintenance, but not the generation, of mammalian neural stem cells. Genes Dev 2002; 16: 846-58.

14. Yamamoto $S$, Nagao M, Sugimori M, et al. Transcription factor expression and notchdependent regulation of neural progenitors in the adult rat spinal cord. J Neurosci 2001; 21: 9814-23. 